\title{
AN ITERATIVE ALGORITHM FOR THE LEAST SQUARES SOLUTIONS OF MATRIX EQUATIONS OVER SYMMETRIC ARROWHEAD MATRICES
}

\author{
Fatemeh Panjeh Ali Beik and Davod Khojasteh Salkuyeh
}

\begin{abstract}
This paper concerns with exploiting an oblique projection technique to solve a general class of large and sparse least squares problem over symmetric arrowhead matrices. As a matter of fact, we develop the conjugate gradient least squares (CGLS) algorithm to obtain the minimum norm symmetric arrowhead least squares solution of the general coupled matrix equations. Furthermore, an approach is offered for computing the optimal approximate symmetric arrowhead solution of the mentioned least squares problem corresponding to a given arbitrary matrix group. In addition, the minimization property of the proposed algorithm is established by utilizing the feature of approximate solutions derived by the projection method. Finally, some numerical experiments are examined which reveal the applicability and feasibility of the handled algorithm.
\end{abstract}

\section{Introduction}

First we introduce the ensuing symbols and notations which are exploited during this paper. The symbols $\operatorname{tr}(A), A^{T}$, Range $(A)$ and $\operatorname{Null}(A)$ are utilized to denote the trace, the transpose, the column space and the null space of the matrix $A$, respectively. The notation $\mathbb{R}^{m \times n}$ stands for the set of all $m \times n$ real matrices. For a given matrix $A$, the symbol $A^{+}$denotes the well-known Moore-Penrose inverse of $A$. A matrix $A \in \mathbb{R}^{n \times n}$ is called a symmetric (skewsymmetric) matrix if $A=A^{T}\left(A=-A^{T}\right)$ and the set of all $n \times n$ symmetric (skew-symmetric) matrices is denoted by $\mathbb{S R}^{n \times n}\left(\mathbb{S S R}^{n \times n}\right)$. The notation $I[1, p]$ refers to the set of all integer numbers between 1 and $p$. Assume that $X=\left(X_{1}, \ldots, X_{q}\right)$ where $X_{j} \in \mathbb{R}^{m_{j} \times n_{j}}$ for $j \in I[1, q]$, we call $X$ as a matrix group.

Linear matrix equations have a cardinal application in numerous fields, such as control theory, system theory, stability theory and some other areas of pure

Received April 18, 2014; Revised January 1, 2015.

2010 Mathematics Subject Classification. Primary 15A24, 65F10.

Key words and phrases. matrix equation, projection technique, iterative algorithm, least squares problem, arrowhead matrix. 
and applied mathematics. Hitherto, several types of iterative algorithms to solve various kinds of matrix equations have been handled in the literature; for further details see $[1,2,10,11,12,15,18,19,26,29,30,34,35,37,38,43]$ and the references therein. In addition the explicit forms of the solutions of some kinds of matrix equations have been derived. For instance, Jiang and Wei [20] have studied the solutions of complex matrix equations $X-A X B=$ $C$ and $X-A \bar{X} B=C$ and obtained explicit solutions of the equations by the method of characteristic polynomial and a method of real representation of a complex matrix, respectively. In [39], Wu et al. have propounded an unified approach to resolve a general class of Sylvester-polynomial-conjugate matrix equations which include the Yakubovich-conjugate matrix equation as a special case. Several explicit parametric solutions to the generalized Sylvesterconjugate matrix equation have been presented in [40].

The idea of conjugate gradient (CG) method [33] has been developed for constructing iterative algorithms to compute the solutions of different kinds of linear matrix equations over generalized reflexive and anti-reflexive, generalized bisymmetric, generalized centro-symmetric, mirror-symmetric, skewsymmetric and $(P, Q)$-reflexive matrices, for more details see $[2,7,8,9,16,22$, 29, 38] and the references therein. For instance, Peng et al. [28] have proposed an iterative algorithm for finding the bisymmetric solutions of matrix equation

$$
A_{1} X_{1} B_{1}+A_{2} X_{2} B_{2}+\cdots+A_{l} X_{l} B_{l}=C .
$$

Recently, the conjugate gradient least squares (CGLS) [33] method has been extended to present iterative algorithms for finding least squares solutions of various kinds of (coupled) matrix equations. For example, Peng [26] has offered an algorithm for minimizing

$$
\left\|A_{1} X_{1} B_{1}+A_{2} X_{2} B_{2}+\cdots+A_{l} X_{l} B_{l}-C\right\|_{F}
$$

where $\|\cdot\|_{F}$ is the Frobenius norm and $X_{j}(j \in I[1, l])$ is a reflexive matrix with a specified central principal submatrix. In [31], Peng and Zhou have offered an algorithm to minimize (1) where $X_{j}(j \in I[1, l])$ is bisymmetric with a specified central principal. In an alternative work, Peng and Xin [30] have extended the algorithm in $[26,31]$ to propose an iterative method for solving the general coupled matrix equations

$$
\sum_{j=1}^{l} A_{i j} X_{j} B_{i j}=C_{i}, \quad i=1,2, \ldots, t
$$

where $X_{j} \in \mathbb{R}^{n_{j} \times n_{j}}(j \in I[1, l])$ is a reflexive matrix with a specified central principal submatrix. In [15], Hajarian has developed the CGLS method to determine the minimum norm solutions of the following general least squares 
problem

$$
\left\|\left(\begin{array}{c}
\sum_{j=1}^{q} A_{1, j} X_{j} B_{1, j} \\
\sum_{j=1}^{q} A_{2, j} X_{j} B_{2, j} \\
\vdots \\
\sum_{j=1}^{q} A_{p, j} X_{j} B_{p, j}
\end{array}\right)-\left(\begin{array}{c}
C_{1} \\
C_{2} \\
\vdots \\
C_{p}
\end{array}\right)\right\|=\min .
$$

In this paper we aim to develop the CGLS method for solving the least squares problem for a class of coupled matrix equations over symmetric arrowhead matrices. The inspiration of presenting the current work is briefly expounded in the second section. Here we would like to comment that the topic of constructing iterative algorithms to find the symmetric arrowhead solutions of coupled matrix equation has not been investigated so far. Beside the earlier refereed comment, some of our utilized manners for analyzing the properties of the CGLS method are different to those employed in [15, 30,31]. More precisely, the applied strategy for demonstrating the minimization property of the proposed algorithm.

Before ending the present section, we recollect some definitions and properties which are used throughout this paper. The inner product of $X, Y \in \mathbb{R}^{m \times n}$ is defined by $\langle X, Y\rangle_{F}=\operatorname{tr}\left(Y^{T} X\right)$. The induced norm is the well-known Frobenius norm, i.e., the norm of $Y \in \mathbb{R}^{m \times p}$ is given by $\|Y\|_{F}=\sqrt{\operatorname{tr}\left(Y^{T} Y\right)}$. It is natural to expound the inner product of the matrix groups $X=\left(X_{1}, \ldots, X_{q}\right)$ and $Y=\left(Y_{1}, \ldots, Y_{q}\right)$ by

$$
\langle X, Y\rangle=\left\langle X_{1}, Y_{1}\right\rangle_{F}+\left\langle X_{2}, Y_{2}\right\rangle_{F}+\cdots+\left\langle X_{q}, Y_{q}\right\rangle_{F}
$$

where $X_{j}, Y_{j} \in \mathbb{R}^{n_{j} \times m_{j}}$ for $j \in I[1, q]$. Therefore we may define the norm of the matrix group $X=\left(X_{1}, \ldots, X_{q}\right)$ as follows:

$$
\|X\|^{2}=\left\|X_{1}\right\|_{F}^{2}+\left\|X_{2}\right\|_{F}^{2}+\cdots+\left\|X_{q}\right\|_{F}^{2},
$$

where $X_{j} \in \mathbb{R}^{n_{j} \times m_{j}}$ for $j \in I[1, q]$.

Suppose that $A=\left[a_{i j}\right]_{m \times s}$ and $B=\left[b_{i j}\right]_{n \times q}$ are real matrices, the Kronecker product of the matrices $A$ and $B$ is defined as the $m n \times s q$ matrix $A \otimes B=\left[a_{i j} B\right]$. The "vec" operator transmutes a matrix $A$ of size $m \times s$ to a vector $a=\operatorname{vec}(A)$ of size $m s \times 1$ via stacking the columns of $A$. The following useful equality can be verified easily (See [3])

$$
\operatorname{vec}(A X B)=\left(B^{T} \otimes A\right) \operatorname{vec}(X) .
$$

Definition $1.1([23])$. A given matrix $A \in \mathbb{R}^{n \times n}$ is called a symmetric arrowhead matrix if it has the following form:

$$
A=\left[\begin{array}{ccccc}
a_{1} & b_{1} & b_{2} & \cdots & b_{n-1} \\
b_{1} & a_{2} & 0 & \cdots & 0 \\
b_{2} & 0 & a_{3} & \cdots & 0 \\
\vdots & \vdots & \vdots & \ddots & \vdots \\
b_{n-1} & 0 & 0 & \cdots & a_{n}
\end{array}\right]
$$


In practice arrowhead matrices occur in numerous areas, see [14, 24, 42]. For instance, when the Lanczos method is exploited for solving the eigenvalue problem for large sparse matrices [25]. In addition, the eigenstructure problems of arrowhead matrices emerge from applications in molecular physics [23]. In [21], it is pointed that the symmetric arrowhead matrices can denote the parameter matrices of the control equations of nonlinear control systems.

The set of all $n \times n$ symmetric arrowhead matrices is represented by $\mathbb{S} \mathbb{R} \mathbb{R}^{n \times n}$. The matrix group $X=\left(X_{1}, X_{2}, \ldots, X_{q}\right)$ is said to be a symmetric arrowhead matrix group if the matrices $X_{1}, X_{2}, \ldots, X_{q}$ are symmetric arrowhead matrices.

The outline of this paper is organized as follows. In the next section we momentarily recount our goal for presenting the current work and state two main problems which this paper is concerned with. In Section 3, we establish some useful theoretical results which capable us to analyze the properties of our proposed algorithm. Section 4 is devoted to elaborating an algorithm for solving the mentioned problems and investigating its convergence properties. Numerical experiments are reported to illustrate the effectively and applicability of the propounded algorithm in Section 5. Finally the paper is finished with a brief conclusion in Section 6 .

\section{Motivation and main contribution}

In $[5,27]$, a specific inverse eigenvalue problem has been studied which consists of constructing the symmetric arrowhead matrix from spectral data. The generalized inverse eigenvalue problem for symmetric arrowhead matrices consists of determining nontrivial symmetric arrowhead matrices $A$ and $B$ such that

$$
A X=\Lambda B X
$$

where $X$ and the diagonal matrix $\Lambda$ are known such that the columns of $X$ are given eigenvectors and the diagonal elements of $\Lambda$ are the given associated eigenvalues. In [41], an approach has been offered to solve the generalized inverse eigenvalue problem (2). Moreover, the author has calculated the nearest solution of (2) to an arbitrary given symmetric arrowhead matrices $\tilde{A}$ and $\tilde{B}$. Nevertheless, it can be easily checked out that the proposed manner has high computational costs.

As seen (2) is a linear matrix equation and it can be a inspiration to investigate about the symmetric arrowhead solutions of matrix equations. For instance, recently, Li et al. [21] have derived the general expression of the least squares solution of the matrix equation $A X B+C Y D=E$ with the least norm for symmetric arrowhead matrices. In fact the authors has considered the following problem.

Problem 2.1. Given $A \in \mathbb{R}^{m \times n}, B \in \mathbb{R}^{n \times s}, C \in \mathbb{R}^{m \times k}, D \in \mathbb{R}^{k \times s}, E \in \mathbb{R}^{m \times s}$, let

$$
\bar{S}_{E}=\left\{[X, Y] \mid X \in \mathbb{S A R}^{n \times n}, Y \in \mathbb{S A}^{k \times k},\|A X B+C Y D-E\|=\min \right\} .
$$


Find out $[\hat{X}, \hat{Y}] \in \bar{S}_{E}$ such that

$$
\|\hat{X}\|_{F}^{2}+\|\hat{Y}\|_{F}^{2}=\min _{[X, Y] \in \bar{S}_{E}}\left(\|X\|_{F}^{2}+\|Y\|_{F}^{2}\right) .
$$

In [21], the Moore-Penrose inverse and the Kronecker product have been exploited to obtain the next algorithm for solving Problem 2.1. Note that in the following algorithm, the matrix $H_{l}(l=n, k)$ has the following structure

$$
H_{l}=\left[\begin{array}{ccccccccccc}
e_{1} & e_{2} & e_{3} & \cdots & e_{l-1} & e_{l} & 0 & 0 & \cdots & 0 & 0 \\
0 & e_{1} & 0 & \cdots & 0 & 0 & e_{2} & 0 & \cdots & 0 & 0 \\
0 & 0 & e_{1} & \cdots & 0 & 0 & 0 & e_{3} & \cdots & 0 & 0 \\
\vdots & \vdots & \vdots & & \vdots & \vdots & \vdots & \vdots & & \vdots & \vdots \\
0 & 0 & 0 & \cdots & e_{1} & 0 & 0 & 0 & \cdots & e_{l-1} & 0 \\
0 & 0 & 0 & \cdots & 0 & e_{1} & 0 & 0 & \cdots & 0 & e_{l}
\end{array}\right],
$$

where $e_{i}$ is a unite vector with the $i$ th entry is one and the others are zero.

Algorithm 1 (Li et al. [21]).

Step 1. Input $A \in \mathbb{R}^{m \times n}, B \in \mathbb{R}^{n \times s}, C \in \mathbb{R}^{m \times k}, D \in \mathbb{R}^{k \times s}, E \in \mathbb{R}^{m \times s}$ and $H_{n}, H_{k}$.

Step 2. Compute $P_{1}=\left(B^{T} \otimes A\right) H_{n}, P_{2}=\left(D^{T} \otimes C\right) H_{k}$.

Step 3. Compute $R, G, Z, K_{11}, K_{12}, K_{22}$ as follows:

$$
\begin{aligned}
R & =\left(I-P_{1} P_{1}^{+}\right) P_{2} \\
G & =R^{+}+\left(I-R^{+} R\right) Z P_{2}^{T}\left(P_{1}^{+}\right)^{T} P_{1}^{+}\left(I-P_{2} R^{+}\right), \\
Z & =\left(I+\left(I-R^{+} R\right) P_{2}^{T}\left(P_{1}^{+}\right)^{T} P_{1}^{+} P_{2}\left(I-R^{+} R\right)\right)^{-1}, \\
K_{11} & =I-P_{1}^{+} P_{1}+P_{1}^{+} P_{2} Z\left(I-R^{+} R\right) P_{2}^{T}\left(P_{1}^{+}\right)^{T}, \\
K_{12} & =-P_{1}^{+} P_{2}\left(I-R^{+} R\right) Z, \\
K_{22} & =\left(I-R^{+} R\right) Z .
\end{aligned}
$$

Step 4. If the equality $\left(P_{1} P_{1}^{+}+R R^{+}\right) \operatorname{vec}(E)=\operatorname{vec}(E)$ holds, then compute $[\hat{X}, \hat{Y}]$ such that $\left[\operatorname{vec}(\hat{X})^{T}, \operatorname{vec}(\hat{Y})^{T}\right]^{T}$ is equal to the following vector

$$
\left(I-H\left[\begin{array}{ll}
K_{11} & K_{12} \\
K_{12}^{T} & K_{22}
\end{array}\right]\left[H\left[\begin{array}{cc}
K_{11} & K_{12} \\
K_{12}^{T} & K_{22}
\end{array}\right]\right]^{+}\right) H\left[\begin{array}{c}
P_{1}^{+}-P_{1}^{+} P_{2} G \\
G
\end{array}\right] \operatorname{vec}(E)
$$

where $H=\operatorname{diag}\left(H_{n}, H_{k}\right)$.

There are mainly two reasons which motivate us to develop an efficient iterative algorithm to solve linear matrix equations over symmetric arrowhead matrices. The first is that Algorithm 1 is too expensive in practice due to exploiting the Kronecker product and requiring the computation of the MoorePenrose inverses. On the other hand, to the best of our knowledge, the subject of applying of an iterative method to resolve coupled linear matrix equations over symmetric arrowhead matrices has not been investigated so far.

In order to derive the algorithm for more general cases, in this paper we consider the following two main problems. 
Problem 2.2. Assume that the matrices $A_{i j \ell} \in \mathbb{R}^{r_{i} \times n_{j}}, B_{i j \ell} \in \mathbb{R}^{r_{i} \times n_{j}}$ and $C_{i} \in \mathbb{R}^{r_{i} \times s_{i}}$ are given for $i \in I[1, N], j \in I[1, q]$ and $\ell \in I[1, p]$. Moreover, suppose that

$S_{E}=\left\{\left(X_{1}, \ldots, X_{q}\right) \mid X_{j} \in \mathbb{S} \mathbb{A} \mathbb{R}^{n_{j} \times n_{j}}, \sum_{i=1}^{N}\left\|\sum_{\ell=1}^{p} \sum_{j=1}^{q} A_{i j \ell} X_{j} B_{i j \ell}-C_{i}\right\|_{F}^{2}=\min \right\}$.

Find the least norm symmetric arrowhead solution group $\hat{X}=\left(\hat{X}_{1}, \ldots, \hat{X}_{q}\right) \in$ $S_{E}$ such that

$$
\|\hat{X}\|=\min _{X \in S_{E}}\|X\| .
$$

Problem 2.3. Assume that $S_{E}$ is defined as (3). For a given arbitrary matrix group $\tilde{X}=\left(\tilde{X}_{1}, \ldots, \tilde{X}_{q}\right)$. Find $X^{*}=\left(X_{1}^{*}, \ldots, X_{q}^{*}\right) \in S_{E}$ such that

$$
\left\|X^{*}-\tilde{X}\right\|=\min _{X \in S_{E}}\|X-\tilde{X}\| \text {. }
$$

For simplicity, we exploit the following linear operator

$$
\begin{aligned}
\mathcal{M}: \mathbb{R}^{n_{1} \times n_{1}} \times \cdots \times \mathbb{R}^{n_{q} \times n_{q}} & \rightarrow \mathbb{R}^{r_{1} \times s_{1}} \times \cdots \times \mathbb{R}^{r_{N} \times s_{N}}, \\
X=\left(X_{1}, \ldots, X_{q}\right) & \mapsto \mathcal{M}(X):=\left(M_{1}(X), \ldots, M_{N}(X)\right),
\end{aligned}
$$

where

$$
M_{i}(X)=\sum_{\ell=1}^{p} \sum_{j=1}^{q} A_{i j \ell} X_{j} B_{i j \ell}, \quad i=1,2, \ldots, N .
$$

In fact we mainly focus on determining the minimum norm symmetric arrowhead solution of the following general least squares problem

$$
\|\mathcal{M}(X)-C\|=\min ,
$$

where $C=\left(C_{1}, \ldots, C_{N}\right)$.

\section{Preliminary theoretical results}

The current section deals with proving some useful theoretical results which capable us to construct and analyze an algorithm for solving Problems 2.2 and 2.3. Meanwhile, some notations are introduced.

Let us consider the linear subspace $\mathbb{C} \mathbb{A} \mathbb{R}^{n \times n}$ on $\mathbb{R}^{n \times n}$ defined as follows:

$$
\mathbb{C S A R}^{n \times n}=\left\{X \mid X=\left[\begin{array}{ccccc}
0 & 0 & 0 & \cdots & 0 \\
0 & 0 & a_{23} & \cdots & a_{2 n} \\
0 & a_{32} & 0 & \ddots & \vdots \\
\vdots & \vdots & \ddots & \ddots & a_{n-1 n} \\
0 & a_{n 2} & \cdots & a_{n n-1} & 0
\end{array}\right] \text { and } X=X^{T}\right\} \text {. }
$$

Now we present an important lemma which demonstrates that $\mathbb{R}^{n \times n}$ can be

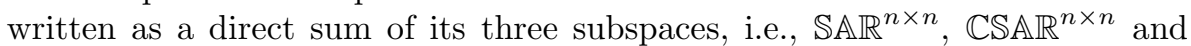
$\mathbb{S S R}^{n \times n}$. 
Lemma 3.1. Assume that the linear subspaces $\mathbb{S} \mathbb{R}^{n \times n}, \mathbb{C} \mathbb{S} \mathbb{R}^{n \times n}$ and $\mathbb{S S R}^{n \times n}$ are defined as before. Then the following statement holds:

$$
\mathbb{R}^{n \times n}=\mathbb{S} \mathbb{A} \mathbb{R}^{n \times n} \oplus \mathbb{C} \mathbb{S} \mathbb{R}^{n \times n} \oplus \mathbb{S} \mathbb{S}^{n \times n} .
$$

Here $\oplus$ refers to the orthogonal direct sum with respect to $\langle\cdot, \cdot\rangle_{F}$.

Proof. It is well-known that

$$
\mathbb{R}^{n \times n}=\mathbb{S R}^{n \times n} \oplus \mathbb{S S R}^{n \times n} .
$$

That is, for an arbitrary $Z \in \mathbb{R}^{n \times n}$ we have

$$
Z=Z_{1}+Z_{2}
$$

where $Z_{1}=\left(Z+Z^{T}\right) / 2, Z_{2}=\left(Z-Z^{T}\right) / 2$ and $\left\langle Z_{1}, Z_{2}\right\rangle_{F}=0$. Evidently the symmetric matrix $Z_{1}=\left[Z_{i j}^{(1)}\right]$ be written as follows:

$$
Z_{1}=Z_{1}^{\prime}+Z_{2}^{\prime}
$$

where $Z_{1}^{\prime} \in \mathbb{S} \mathbb{A} \mathbb{R}^{n \times n}$ and $Z_{2}^{\prime} \in \mathbb{C} \mathbb{A} \mathbb{R}^{n \times n}$ are respectively defined by

$$
Z_{1}^{\prime}=\left[\begin{array}{ccccc}
Z_{11}^{(1)} & Z_{12}^{(1)} & Z_{13}^{(1)} & \ldots & Z_{1 n}^{(1)} \\
Z_{21}^{(1)} & Z_{22}^{(1)} & 0 & \ldots & 0 \\
Z_{31}^{(1)} & 0 & Z_{33}^{(1)} & \ldots & 0 \\
\vdots & \vdots & \vdots & \ddots & \vdots \\
Z_{n 1}^{(1)} & 0 & 0 & \cdots & Z_{n n}^{(1)}
\end{array}\right]
$$

and $Z_{2}^{\prime}=Z_{1}-Z_{1}^{\prime}$. It is not difficult to verify that

$$
\left\langle Z_{1}^{\prime}, Z_{2}^{\prime}\right\rangle_{F}=\operatorname{tr}\left(\left(Z_{1}^{\prime}\right)^{T} Z_{2}^{\prime}\right)=0 .
$$

From the above results, it can be deduced that $\mathbb{S R}^{n \times n}=\mathbb{S} \mathbb{A} \mathbb{R}^{n \times n} \oplus \mathbb{C} \mathbb{A} \mathbb{R}^{n \times n}$. On the other hand, the matrix $Z_{1}^{\prime}$ is symmetric and $Z_{2}$ is a skew-symmetric matrix which imply that

$$
\begin{aligned}
\left\langle Z_{1}^{\prime}, Z_{2}\right\rangle_{F} & =\operatorname{tr}\left(Z_{2}^{T} Z_{1}^{\prime}\right) \\
& =-\operatorname{tr}\left(Z_{2} Z_{1}^{\prime}\right)=-\operatorname{tr}\left(Z_{1}^{\prime} Z_{2}\right) \\
& =-\operatorname{tr}\left(\left(Z_{1}^{\prime}\right)^{T} Z_{2}\right)=-\left\langle Z_{2}, Z_{1}^{\prime}\right\rangle_{F}=-\left\langle Z_{1}^{\prime}, Z_{2}\right\rangle_{F} .
\end{aligned}
$$

Therefore $\left\langle Z_{1}^{\prime}, Z_{2}\right\rangle_{F}=0$. As $Z_{2}^{\prime}$ is a symmetric matrix, we may show that $\left\langle Z_{2}^{\prime}, Z_{2}\right\rangle_{F}=0$ in a similar manner. Now the result can be concluded immediately.

In what follows we consider the linear operator $\mathcal{A}$ expounded by

$$
\begin{aligned}
\mathcal{A}: \mathbb{R}^{n_{1} \times n_{1}} \times \cdots \times \mathbb{R}^{n_{q} \times n_{q}} & \rightarrow \mathbb{R}^{n_{1} \times n_{1}} \times \cdots \times \mathbb{R}^{n_{q} \times n_{q}}, \\
X=\left(X_{1}, \ldots, X_{q}\right) & \mapsto \mathcal{A}(X):=\left(\frac{\mathcal{A}_{1}+\mathcal{A}_{1}^{T}}{2}, \ldots, \frac{\mathcal{A}_{q}+\mathcal{A}_{q}^{T}}{2}\right),
\end{aligned}
$$


where $\mathcal{A}_{l}$ corresponding to $X_{l}=\left[X_{i j}^{(l)}\right]_{n_{j} \times n_{j}}$ is elucidated by

$$
\mathcal{A}_{l}=\left[\begin{array}{ccccc}
X_{11}^{(l)} & X_{12}^{(l)} & X_{13}^{(l)} & \cdots & X_{1 n}^{(l)} \\
X_{21}^{(l)} & X_{22}^{(l)} & 0 & \cdots & 0 \\
X_{31}^{(l)} & 0 & X_{33}^{(l)} & \cdots & 0 \\
\vdots & \vdots & \vdots & \ddots & \vdots \\
X_{n 1}^{(l)} & 0 & 0 & \cdots & X_{n n}^{(l)}
\end{array}\right], \quad l \in I[1, q] .
$$

Now we establish the following proposition which provides the effective tools to scrutinize the properties of the sequence of approximate solutions produced by the presented main algorithm.

Proposition 3.2. Suppose that the linear operators $\mathcal{A}$ and $\mathcal{M}$ are defined as before. Let $P=\left(P_{1}, \ldots, P_{q}\right)$ be an arbitrary symmetric arrowhead matrix group. Moreover assume that the linear operator $\mathcal{M}^{*}$ is given by

$$
\begin{aligned}
\mathcal{M}^{*}: \mathbb{R}^{r_{1} \times s_{1}} \times \cdots \times \mathbb{R}^{r_{N} \times s_{N}} & \rightarrow \mathbb{R}^{n_{1} \times n_{1}} \times \cdots \times \mathbb{R}^{n_{q} \times n_{q}}, \\
Y=\left(Y_{1}, \ldots, Y_{N}\right) & \mapsto \mathcal{M}^{*}(Y):=\left(M_{1}^{*}(Y), \ldots, M_{q}^{*}(Y)\right),
\end{aligned}
$$

where

$$
M_{j}^{*}(Y)=\sum_{\ell=1}^{p} \sum_{i=1}^{N} A_{i j \ell}^{T} Y_{i} B_{i j \ell}^{T}, \quad j \in I[1, q] .
$$

Then for arbitrary given matrix groups $X=\left(X_{1}, \ldots, X_{q}\right)$ and $Y=\left(Y_{1}, \ldots, Y_{p}\right)$, the following statements hold

$$
\begin{aligned}
\langle\mathcal{M}(X), Y\rangle & =\left\langle X, \mathcal{M}^{*}(Y)\right\rangle, \\
\left\langle P, \mathcal{M}^{*}(Y)\right\rangle & =\left\langle P, \mathcal{A}\left(\mathcal{M}^{*}(Y)\right)\right\rangle, \\
\langle\mathcal{M}(P), Y\rangle & =\left\langle P, \mathcal{A}\left(\mathcal{M}^{*}(Y)\right)\right\rangle .
\end{aligned}
$$

Proof. It is known that for given arbitrary matrices $A, B$, we have $\operatorname{tr}(A B)=$ $\operatorname{tr}\left(A^{T} B^{T}\right)=\operatorname{tr}(B A)$. Now straightforward computations turn out that

$$
\begin{aligned}
\langle\mathcal{M}(X), Y\rangle & =\sum_{i=1}^{N} \operatorname{tr}\left(Y_{i}^{T}\left(\sum_{\ell=1}^{p} \sum_{j=1}^{q} A_{i j \ell} X_{j} B_{i j \ell}\right)\right) \\
& =\operatorname{tr}\left(\sum_{i=1}^{N} \sum_{\ell=1}^{p} \sum_{j=1}^{q} Y_{i}^{T}\left(A_{i j \ell} X_{j} B_{i j \ell}\right)\right) \\
& =\sum_{i=1}^{N} \sum_{\ell=1}^{p} \sum_{j=1}^{q} \operatorname{tr}\left(Y_{i} B_{i j \ell}^{T} X_{j}^{T} A_{i j \ell}^{T}\right) \\
& =\sum_{i=1}^{N} \sum_{\ell=1}^{p} \sum_{j=1}^{q} \operatorname{tr}\left(A_{i j \ell}^{T} Y_{i} B_{i j \ell}^{T} X_{j}^{T}\right),
\end{aligned}
$$




$$
\begin{aligned}
& =\sum_{j=1}^{q} \operatorname{tr}\left(X_{j}^{T}\left(\sum_{i=1}^{N} \sum_{\ell=1}^{p} A_{i j \ell}^{T} Y_{i} B_{i j \ell}^{T}\right)\right) \\
& =\left\langle X, \mathcal{M}^{*}(Y)\right\rangle .
\end{aligned}
$$

The relation (6) can be concluded from Lemma 3.1. The last equality follows from Eqs. (5) and (6).

The following lemma known as the Projection Theorem which is useful for obtaining the next results in this paper, the proof of theorem can be found in [36].

Lemma 3.3. Let $\mathbb{W}$ be a finite dimensional space, $\mathbb{U}$ be a subspace of $\mathbb{W}$, and $\mathbb{U}^{\perp}$ be the orthogonal complement subspace of $\mathbb{U}$. For a given $w \in \mathbb{W}$, always, there exists $u_{0} \in \mathbb{U}$ such that $\left\|w-u_{0}\right\| \leq\|w-u\|$ for any $u \in \mathbb{U}$. More precisely, $u_{0} \in \mathbb{U}$ is the unique minimization vector in $\mathbb{U}$ if and only if $\left(w-u_{0}\right) \perp \mathbb{U}$. Here $\|\cdot\|$ is the norm corresponding to the inner product defined on $\mathbb{W}$.

The following proposition is a direct conclusion of Proposition 3.2 and Lemma 3.3.

Proposition 3.4. The symmetric arrowhead matrix group $\hat{X}=\left(\hat{X}_{1}, \ldots, \hat{X}_{q}\right)$ is the solution of Problem 2.2 if and only if

$$
\left\langle\mathcal{M}^{*}(C-\mathcal{M}(\hat{X})), X\right\rangle=0
$$

or equivalently,

$$
\left\langle\mathcal{A}\left(\mathcal{M}^{*}(\hat{R})\right), X\right\rangle=0
$$

where $\hat{R}=C-\mathcal{M}(\hat{X})$ and $X=\left(X_{1}, \ldots, X_{q}\right)$ is an arbitrary given symmetric arrowhead matrix group.

Proof. By Lemma 3.3, $\hat{X}=\left(\hat{X}_{1}, \ldots, \hat{X}_{q}\right)$ is the solution of Problem 2.2 if and only if

$$
C-\mathcal{M}(\hat{X}) \perp \mathcal{M}(X)
$$

for any symmetric arrowhead matrix group $X=\left(X_{1}, \ldots, X_{q}\right)$, i.e.,

$$
\langle C-\mathcal{M}(\hat{X}), \mathcal{M}(X)\rangle=0 \text {. }
$$

Now the results follow immediately from Proposition 3.2.

The following remark of the above proposition provides a sufficient condition for $\hat{X}=\left(\hat{X}_{1}, \ldots, \hat{X}_{q}\right)$ to be the solution of Problem 2.2.

Remark 3.5. Suppose that $\hat{X}=\left(\hat{X}_{1}, \ldots, \hat{X}_{q}\right)$ is a symmetric arrowhead matrix group and $\hat{R}=C-\mathcal{M}(\hat{X})=\left(\hat{R}_{1}, \ldots, \hat{R}_{N}\right)$. If

$$
\mathcal{A}\left(\mathcal{M}^{*}(\hat{R})\right)=0,
$$

then Proposition 3.4 implies that $\hat{X}$ is the solution of Problem 2.2. 
Proposition 3.6. Suppose that $\hat{X}$ is the solution of Problem 2.2 and the set $S_{E}$ is determined by (3). Then the following statements hold

(1) Assume that $\tilde{X}=\hat{X}+\bar{X}$ where $\bar{X}$ is a given symmetric arrowhead matrix group such that $\mathcal{M}(\bar{X})=0$. Then $\tilde{X} \in S_{E}$.

(2) Suppose that $\tilde{X} \in S_{E}$, then $\mathcal{M}(\tilde{X}-\hat{X})=0$, i.e., there exists a symmetric arrowhead matrix group $\bar{X}$ such that $\bar{X}=\tilde{X}-\hat{X}$ and $\mathcal{M}(\bar{X})=0$.

Proof. Suppose that $\bar{X}$ is a given symmetric arrowhead matrix group such that $\mathcal{M}(\bar{X})=0$. Let $\hat{X}$ be the solution of Problem 2.2 and $\tilde{X}=\hat{X}+\bar{X}$. Evidently,

$$
\begin{aligned}
\langle C-\mathcal{M}(\tilde{X}), C-\mathcal{M}(\tilde{X})\rangle & =\langle C-\mathcal{M}(\hat{X})-\mathcal{M}(\bar{X}), C-\mathcal{M}(\hat{X})-\mathcal{M}(\bar{X})\rangle \\
& =\langle C-\mathcal{M}(\hat{X}), C-\mathcal{M}(\hat{X})\rangle .
\end{aligned}
$$

The preceding equality together with the fact that $\bar{X} \in \mathbb{S} A \mathbb{R}^{n \times n}$ reveal that $\tilde{X} \in S_{E}$ which proves the first part.

Now we establish the second part. Suppose that $\tilde{X} \in S_{E}$ and without loss of generality assume that $\tilde{X} \neq \hat{X}$. As $\hat{X}, \tilde{X} \in S_{E}$, we may conclude that

$$
\langle C-\mathcal{M}(\tilde{X}), C-\mathcal{M}(\tilde{X})\rangle=\langle C-\mathcal{M}(\hat{X}), C-\mathcal{M}(\hat{X})\rangle .
$$

Note that with an analogous explanation for concluding (8), we may deduce that

$$
\langle C-\mathcal{M}(\hat{X}), \mathcal{M}(\tilde{X}-\hat{X})\rangle=0 .
$$

By some easy computations, we derive that

$$
\begin{aligned}
\langle C-\mathcal{M}(\tilde{X}), C-\mathcal{M}(\tilde{X})\rangle= & \langle C-\mathcal{M}(\hat{X}-(\tilde{X}-\hat{X})), C-\mathcal{M}(\hat{X}-(\tilde{X}-\hat{X}))\rangle \\
= & \langle C-\mathcal{M}(\hat{X}), C-\mathcal{M}(\hat{X})\rangle \\
& -2\langle C-\mathcal{M}(\hat{X}), \mathcal{M}(\tilde{X}-\hat{X})\rangle \\
& +\langle\mathcal{M}(\tilde{X}-\hat{X}), \mathcal{M}(\tilde{X}-\hat{X})\rangle
\end{aligned}
$$

In view of (10) and (11), the above relation implies that

$$
\langle\mathcal{M}(\tilde{X}-\hat{X}), \mathcal{M}(\tilde{X}-\hat{X})\rangle=0,
$$

which completes the proof.

\section{An algorithm and its analysis}

The conjugate gradient least squares (CGLS) method is an efficient algorithm for solving the large sparse least squares problem

$$
\min _{x \in \mathbb{R}^{n}}\|b-A x\|_{2},
$$


where $\|\cdot\|_{2}$ is the well-known Euclidean vector norm, $A \in \mathbb{R}^{m \times n}$ and $b \in \mathbb{R}^{m}$ are known; for further details see $[4,33]$.

In this section we develop the CGLS method to construct an iterative algorithm for solving Problems 2.2 and 2.3.

\subsection{An iterative algorithm to resolve Problem 2.2}

In the following, we present an extended form of the CGLS method for finding the solution of the first problem.

Algorithm 2. An iterative algorithm for solving Problem 2.2

1. Choose an arbitrary symmetric arrowhead matrix group $X(0)$ and a tolerance $\epsilon$. (For simplicity we may set $X(0)=0$.)

2. Set $k=0$ and compute

- $R(0)=C-\mathcal{M}(X(0))$

- $P(0)=\mathcal{A}\left(\mathcal{M}^{*}(R(0))\right.$

- $Q(0)=P(0)$.

3. If $\|P(k)\|<\epsilon$ or $\|R(k)\|<\epsilon$, then stop else goto the next step.

4. Calculate

- $X(k+1)=X(k)+\frac{\|P(k)\|^{2}}{\|\mathcal{M}(Q(k))\|^{2}} Q(k)$;

- $R(k+1)=R(k)-\frac{\|P(k)\|^{2}}{\|\mathcal{M}(Q(k))\|^{2}} \mathcal{M}(Q(k))$;

- $P(k+1)=\mathcal{A}\left(\mathcal{M}^{*}(R(k+1))\right)$

- $Q(k+1)=P(k+1)+\frac{\|P(k+1)\|^{2}}{\|P(k)\|^{2}} Q(k)$;

- $k:=k+1$.

5. Goto Step 3.

Remark 4.1. We would like to point out here that

(1) Evidently at each iterate the matrix groups $P(k)$ and $Q(k)$ are symmetric arrowhead matrix groups. Hence the approximate solution $X(k)$ at each iterate is a symmetric arrowhead matrix group.

(2) If $R(k)=0$, then the $k$ th approximate solution $X(k)$ is the exact solution of the coupled matrix equations $\mathcal{M}(X)=C$ over the symmetric arrowhead matrices.

Theorem 4.2. Suppose that $k$ steps of Algorithm 2 have been performed, i.e., $P(l) \neq 0$ and $\mathcal{M}(Q(l)) \neq 0$ for $l=0,1, \ldots, k$. The sequences $P(l)$ and $Q(l)$ $(l=0,1, \ldots, k)$ produced by Algorithm 2 satisfy

(1) $\langle P(i), P(j)\rangle=0$

(2) $\langle\mathcal{M}(Q(i)), \mathcal{M}(Q(j))\rangle=0$,

(3) $\langle Q(i), P(j)\rangle=0$,

for $i, j=0,1,2, \ldots, k(i \neq j)$.

The proof of Theorem 4.2 is given in Appendix A. In the following remark, we will discuss the assumption $P(l) \neq 0$ and $\mathcal{M}(Q(l)) \neq 0(l=0,1, \ldots, k)$ in 
the previous theorem. More precisely, we clarify that if there exists $t$ such that $P(t)=0$ or $\mathcal{M}(Q(t))=0$, then $X(t)$ is the exact solution of Problem 2.2.

Remark 4.3. Suppose that $t(t \geq 1)$ steps of Algorithm 2 have been performed, i.e., $P(l) \neq 0$ and $\mathcal{M}(Q(l)) \neq 0$ for $l=0,1, \ldots, t-1$.

(1) Consider the case that $P(t)=0$ but $R(k) \neq 0$. By Remark 3.5, $P(t)=$ 0 implies that $X(t)$ is the solution of Problem 2.2.

(2) Now let us consider the case that $\mathcal{M}(Q(t))=0$. Note that $Q(t)$ is a symmetric arrowhead matrix group. Therefore, using Proposition 3.2 we deduce that

$$
\begin{aligned}
0 & =\langle\mathcal{M}(Q(t)), R(t)\rangle \\
& =\left\langle Q(t), \mathcal{M}^{*}(R(t))\right\rangle \\
& =\langle Q(t), P(t)\rangle \\
& =\left\langle P(t)+\frac{\|P(t)\|^{2}}{\|P(t-1)\|^{2}} Q(t-1), P(t)\right\rangle \\
& =\langle P(t), P(t)\rangle+\frac{\|P(t)\|^{2}}{\|P(t-1)\|^{2}}\langle Q(t-1), P(t)\rangle .
\end{aligned}
$$

Now Theorem 4.2 implies that $\langle P(t), P(t)\rangle=0$ which is equivalent to say that $P(t)=0$. That is $\mathcal{M}(Q(t))=0$ indicates that $X(t)$ is the solution of Problem 2.2.

The following theorem turns out that the solution of Problem 2.2 can be computed by Algorithm 2 within finite number of steps for any initial symmetric arrowhead matrix group in the exact arithmetic.

Theorem 4.4. In the absence of roundoff errors, the solution of Problem 2.2 can be computed by Algorithm 2 within at most $m+1$ iteration steps where $m=\nu_{1}+\cdots+\nu_{q}$ and $\nu_{j}$ stands for the dimension of the linear subspace $\mathbb{S} \mathbb{A} \mathbb{R}^{n_{j} \times n_{j}}$

Proof. Presume that $P(l) \neq 0$ for $l=0,1, \ldots, m-1$. Therefore, $X(m)$ can be obtained by Algorithm 2. Suppose that $\mathcal{S}$ denotes the matrix subspace consists of all matrices of the form $E=\left(E_{1}, \ldots, E_{q}\right)$ where $E_{i} \in \mathbb{S} \mathbb{A} \mathbb{R}^{n_{i} \times n_{i}}$ for $i=1,2, \ldots, q$. As established by Theorem 4.2, we have

$$
\langle P(i), P(j)\rangle=0 \quad \text { for } \quad i, j=0,1,2, \ldots, m-1, \quad(i \neq j),
$$

which reveals that $\{P(0), P(1), \ldots, P(m-1)\}$ is an orthogonal basis for $\mathcal{S}$. It is not difficult to see that $P(m)=0$, hence $X(m)$ is the solution of Problem 2.2 .

Consider the least squares problem (12). It is known that the solutions of the least squares problem (12) are computed by $x=A^{+} b+\operatorname{Null}(A)[3]$. Hence, $x^{*}=$ $A^{+} b$ gives the minimum norm solution. Invoking the fact that $\operatorname{Range}\left(A^{+}\right)=$ 
Range $\left(A^{T}\right)$, it is concluded that $x^{*} \in \operatorname{Range}\left(A^{T}\right)$ has the smallest 2-norm solution of the linear system $A x=b$. On the other hand we have

$$
\begin{aligned}
\|C-\mathcal{M}(X)\|^{2} & =\sum_{i=1}^{N}\left\|\sum_{\ell=1}^{p} \sum_{j=1}^{q} A_{i j \ell} X_{j} B_{i j \ell}-C_{i}\right\|_{F}^{2} \\
& =\sum_{i=1}^{N}\left\|\sum_{\ell=1}^{p} \sum_{j=1}^{q}\left(B_{i j \ell}^{T} \otimes A_{i j \ell}\right) \operatorname{vec}\left(X_{j}\right)-\operatorname{vec}\left(C_{i}\right)\right\|_{2}^{2} \\
& =\|\hat{A} x-\hat{c}\|_{2}^{2},
\end{aligned}
$$

where

$$
\hat{A}=\left[\begin{array}{ccc}
\sum_{\ell=1}^{p} B_{11 \ell}^{T} \otimes A_{11 \ell} & \cdots & \sum_{\ell=1}^{p} B_{1 q \ell}^{T} \otimes A_{1 q \ell} \\
\vdots & \ddots & \vdots \\
\sum_{\ell=1}^{p} B_{N 1 \ell}^{T} \otimes A_{N 1 \ell} & \cdots & \sum_{\ell=1}^{p} B_{N q \ell}^{T} \otimes A_{N q \ell}
\end{array}\right], \quad x=\left[\begin{array}{c}
\operatorname{vec}\left(X_{1}\right) \\
\vdots \\
\operatorname{vec}\left(X_{q}\right)
\end{array}\right],
$$

For an arbitrary matrix group $W=\left[W_{1}, \ldots, W_{N}\right]$, it is not difficult to see that the matrix group $\operatorname{vec}(\tilde{X})=\operatorname{vec}\left(\mathcal{M}^{*}(W)\right)$ belongs to $\operatorname{Range}\left(\hat{A}^{T}\right)$. We can deduce that if $X(0)=\mathcal{A}\left(\mathcal{M}^{*}(W)\right)$, then the sequence of approximate solutions produced by Algorithm 2 converges to the least norm solution of the following the least squares problem

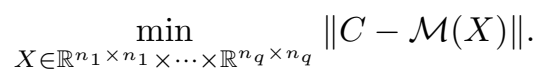

That is Algorithm 2 converges to the solution of Problem 2.2.

\subsection{Minimization property of Algorithm 2}

In order to demonstrate the minimization property of Algorithm 2, we need to establish the following proposition.

Proposition 4.5. Suppose that $k$ steps of Algorithm 2 have been performed, i.e., $P(l) \neq 0$ and $\mathcal{M}(Q(l)) \neq 0$ for $l=0,1, \ldots, k-1$. Then the $k$ th residual $R(k)$ satisfies

$$
\langle R(k), \mathcal{M}(Q(i))\rangle=0, \quad i=0,1, \ldots, k-1 .
$$

Proof. It is known that $Q(i)$ is a symmetric arrowhead matrix group for $i=$ $0,1, \ldots, k-1$. Now by Proposition 3.2 and Theorem 4.2 , it reveals that

$$
\begin{aligned}
\langle R(k), \mathcal{M}(Q(i))\rangle & =\left\langle\mathcal{M}^{*}(R(k)), Q(i)\right\rangle=\left\langle\mathcal{A}\left(\mathcal{M}^{*}(R(k))\right), Q(i)\right\rangle \\
& =\langle P(k), Q(i)\rangle=0 .
\end{aligned}
$$


Assume that $m$ steps of Algorithm 2 have been performed, i.e., $P(l) \neq 0$ and $\mathcal{M}(Q(l)) \neq 0$ for $l=0,1, \ldots, m-1$. Let us define the subspaces $\mathcal{K}_{m}$ and $\mathcal{M K}_{m}$ as follows:

$$
\begin{aligned}
\mathcal{K}_{m} & =\operatorname{span}\{Q(0), \ldots, Q(m-1)\}, \\
\mathcal{M K}_{m} & =\operatorname{span}\{\mathcal{M}(Q(0)), \ldots, \mathcal{M}(Q(m-1))\} .
\end{aligned}
$$

Now in view of Proposition 4.5, it reveals that Algorithm 2 is an oblique projection technique onto $X(0)+\mathcal{K}_{m}\left(X(m) \in X(0)+\mathcal{K}_{m}\right)$ with the orthogonality condition $R(m) \perp \mathcal{M K}_{m}$ where $R(m)=C-\mathcal{M}(X(m))$. The following theorem shows that the residual matrices associated with the approximate solutions produced by Algorithm 2 satisfy an optimality property.

Theorem 4.6 (The minimization property of Algorithm 2). Suppose that $m$ steps of Algorithm 2 have been performed, i.e., $P(l) \neq 0$ and $\mathcal{M}(Q(l)) \neq 0$ for $l=0,1, \ldots, m-1$. Moreover presume that $X(0)$ is an arbitrary given symmetric arrowhead matrix group. Presume that the subspaces $\mathcal{K}_{m}$ and $\mathcal{M K}_{m}$ are defined by (15). Assume that $P(m) \neq 0$, then the $m$-th approximate solution obtained by Algorithm 2 satisfies

$$
\|C-\mathcal{M}(X(m))\|=\min _{X \in X(0)+\mathcal{K}_{m}}\|C-\mathcal{M}(X)\| .
$$

Proof. It is not difficult to see that $X(m) \in X(0)+\mathcal{K}_{m}$. Consequently, there exist $\gamma_{0}^{(m)}, \gamma_{1}^{(m)}, \ldots, \gamma_{m-1}^{(m)}$ such that

$$
X(m)=X(0)+\sum_{i=0}^{m-1} \gamma_{i}^{(m)} Q(i) .
$$

Suppose that $X$ is an arbitrary member of $X(0)+\mathcal{K}_{m}$. That is there exist $\gamma_{0}, \gamma_{1}, \ldots, \gamma_{m-1}$ so that

$$
X=X(0)+\sum_{i=0}^{m-1} \gamma_{i} Q(i)
$$

Assume that $R(m)=C-\mathcal{M}(X(m))$ and $R(X)=C-\mathcal{M}(X)$, it is not onerous to see that

$$
R(X)=R(m)+\sum_{i=0}^{m-1}\left(\gamma_{i}^{(m)}-\gamma_{i}\right) \mathcal{M}(Q(i))
$$

Thus

$$
\begin{aligned}
\langle R(X), R(X)\rangle= & \langle R(m), R(m)\rangle-2 \sum_{i=0}^{m-1}\left(\gamma_{i}^{(m)}-\gamma_{i}\right)\langle\mathcal{M}(Q(i)), R(m)\rangle \\
& +\left\langle\sum_{i=0}^{m-1}\left(\gamma_{i}^{(m)}-\gamma_{i}\right) \mathcal{M}(Q(i)), \sum_{i=0}^{m-1}\left(\gamma_{i}^{(m)}-\gamma_{i}\right) \mathcal{M}(Q(i))\right\rangle .
\end{aligned}
$$


In view of Theorem 4.2 and Proposition 4.5, the above relation reduces to

$$
\langle R(X), R(X)\rangle=\langle R(m), R(m)\rangle+\sum_{i=0}^{m-1}\left(\gamma_{i}^{(m)}-\gamma_{i}\right)^{2}\langle\mathcal{M}(Q(i)), \mathcal{M}(Q(i))\rangle,
$$

which completes the proof.

\subsection{On the solution of Problem 2.3}

Let $\tilde{X}=\left(\tilde{X}_{1}, \ldots, \tilde{X}_{q}\right)$ be a given arbitrary matrix group. By means of Lemma 3.1, we have

$$
\tilde{X}=W_{1}+W_{2}+W_{3},
$$

where $W_{1}=\left(W_{1}^{(1)}, \ldots, W_{q}^{(1)}\right), W_{2}=\left(W_{1}^{(2)}, \ldots, W_{q}^{(2)}\right), W_{3}=\left(W_{1}^{(3)}, \ldots, W_{q}^{(3)}\right)$ such that $W_{j}^{(1)} \in \mathbb{S A R}^{n_{j} \times n_{j}}, W_{j}^{(2)} \in \mathbb{C} \mathbb{A}^{n_{j} \times n_{j}}$ and $W_{j}^{(3)} \in \mathbb{S S R}^{n_{j} \times n_{j}}$ for $j \in I[1, q]$. As a matter of fact $W_{1}=\mathcal{A}(\tilde{X})$ and Lemma 3.1 implies

$$
\left\langle W_{1}-X, W_{2}+W_{3}\right\rangle=0,
$$

where $X=\left(X_{1}, \ldots, X_{q}\right)$ is a symmetric arrowhead solution group. Therefore, it is not difficult to show that

$$
\begin{aligned}
\langle\tilde{X}-X, \tilde{X}-X\rangle= & \left\langle\left(W_{1}-X\right)+\left(W_{2}+W_{3}\right),\left(W_{1}-X\right)+\left(W_{2}+W_{3}\right)\right\rangle \\
= & \left\langle W_{1}-X, W_{1}-X\right\rangle+2\left\langle W_{1}-X, W_{2}+W_{3}\right\rangle \\
& +\left\langle W_{2}+W_{3}, W_{2}+W_{3}\right\rangle \\
= & \left\langle W_{1}-X, W_{1}-X\right\rangle+\left\langle W_{2}+W_{3}, W_{2}+W_{3}\right\rangle .
\end{aligned}
$$

Consequently, we deduce that

$$
\min _{X \in S_{E}}\|X-\tilde{X}\|=\min _{X \in S_{E}}\left\|X-W_{1}\right\| .
$$

As a result, we may apply Algorithm 2 to find the least norm solution of the following least squares problem

$$
\min _{Y \in \hat{S}_{E}}\|\mathcal{M}(Y)-\hat{C}\|
$$

where

$$
\hat{S}_{E}=\left\{Y=\left(Y_{1}, \ldots, Y_{q}\right) \mid Y_{j} \in \mathbb{S} \mathbb{A} \mathbb{R}^{n_{j} \times n_{j}} \text { for } j \in I[1, q]\right\}
$$

and $\hat{C}=C-\mathcal{M}\left(W_{1}\right)$. To determine the solution of Problem 2.3, we first compute $Y^{*}$ as the least norm solution of (16) using the approach described at the end of Subsection 4.1. Then the solution of Problem 2.3 is derived by $X^{*}=Y^{*}+W_{1}$. 


\section{Numerical experiments}

In this section, some numerical experiments are reported to verify the validity of the presented theoretical results and the applicability of Algorithm 2. The execution of all numerical tests was performed in double precision with some Matlab codes on a Pentium 4 PC, with a $3.06 \mathrm{GHz} \mathrm{CPU}$ and 1.00GB of RAM.

Example 5.1. Consider the following two-dimensional convection diffusion equation

$$
\left\{\begin{aligned}
-\Delta u+2 \alpha_{1} u_{x}+2 \alpha_{2} u_{y}-2 \alpha_{3} u & =f, & & \text { in } \Omega, \\
u & =0, & & x \text { on } \partial \Omega,
\end{aligned}\right.
$$

where $\Omega=[0,1] \times[0,1]$ and $\partial \Omega$ is the boundary of $\Omega$. We assume that $\alpha_{1}, \alpha_{2}$ and $\alpha_{3}$ are nonnegative parameters. Discretization of the Laplacian operator by the standard five-point stencil and the first-order derivatives by centered finite differences with mesh size $h=1 /(n+1)$ in both $x$ - and $y$-directions yields the Sylvester matrix equation

$$
A X+X B=C,
$$

where $A$ and $B$ are $n \times n$ matrices of the form

$$
\begin{aligned}
& A=\operatorname{tridiag}\left(-1-\alpha_{1} h, 2-\alpha_{3} h^{2},-1+\alpha_{1} h\right), \\
& B=\operatorname{tridiag}\left(-1-\alpha_{2} h, 2-\alpha_{3} h^{2},-1+\alpha_{2} h\right) .
\end{aligned}
$$

The Sylvester matrix equation (17) with the above data have been examined by several authors so far, for instance see $[6,13,17,32]$. The right-hand side matrix $C$ is taken such a way that the exact solution of (17) is the following symmetric arrowhead matrix

$$
X^{*}=\left[\begin{array}{ccccc}
1 & 1 & 2 & \cdots & n-1 \\
1 & 2 & 0 & \cdots & 0 \\
2 & 0 & 3 & \cdots & 0 \\
\vdots & \vdots & \vdots & \ddots & \vdots \\
n-1 & 0 & 0 & \cdots & n
\end{array}\right] .
$$

We apply Algorithm 2 to solve system (17). The initial guess is set to be $X(0)=0$ and the succeeding stopping criterion is exploited,

$$
E_{k}=\frac{\|R(k)\|_{\infty}}{\|R(0)\|_{\infty}}<10^{-9} .
$$

Numerical results for different values of $n(=1000,2000,3000,4000,5000)$ and three set of parameters $\left(\alpha_{1}, \alpha_{2}, \alpha_{3}\right)=(10,20,10),\left(\alpha_{1}, \alpha_{2}, \alpha_{3}\right)=(50,100,50)$ and $\left(\alpha_{1}, \alpha_{2}, \alpha_{3}\right)=(100,100,0)$ are given in Table 1 . In this table, the number of iterations for the convergence (Iters), CPU times (in seconds) and $E_{k}$ are presented. As seen the proposed algorithm is effectual for computing the symmetric arrowhead solution of (17). 
TABLE 1. Numerical results for Example 5.1 for different values of $n$ and $\left(\alpha_{1}, \alpha_{2}, \alpha_{3}\right)$.

\begin{tabular}{|c|c|c|c|c|c|}
\hline & & $\alpha_{1}=10$ & $\alpha_{2}=20$ & $\alpha_{3}=10$ & \\
\hline & $n=1000$ & $n=2000$ & $n=3000$ & $n=4000$ & $n=5000$ \\
\hline Iters & 25 & 24 & 22 & 22 & 22 \\
\hline$E_{k}$ & $7.6 \mathrm{e}-10$ & $7.0 \mathrm{e}-10$ & $8.8 \mathrm{e}-10$ & $6.3 \mathrm{e}-10$ & $5.0 \mathrm{e}-10$ \\
\hline CPU times & 0.42 & 1.20 & 2.20 & 4.34 & 7.17 \\
\hline
\end{tabular}

\begin{tabular}{|c|c|c|c|c|c|}
\hline & & $\alpha_{1}=50$ & $\alpha_{2}=100$ & $\alpha_{3}=50$ & \\
\hline & $n=1000$ & $n=2000$ & $n=3000$ & $n=4000$ & $n=5000$ \\
\hline Iters & 25 & 24 & 22 & 22 & 22 \\
\hline$E_{k}$ & $8.2 \mathrm{e}-10$ & 7.3e-10 & $9.1 \mathrm{e}-10$ & $6.5 \mathrm{e}-10$ & $5.1 \mathrm{e}-10$ \\
\hline CPU times & 0.42 & 1.23 & 2.22 & 4.27 & 7.09 \\
\hline
\end{tabular}

\begin{tabular}{|c|c|c|c|c|c|}
\hline & & $\alpha_{1}=100$, & $\alpha_{2}=100$, & $\alpha_{3}=0$ & \\
\hline & $n=1000$ & $n=2000$ & $n=3000$ & $n=4000$ & $n=5000$ \\
\hline Iters & 25 & 24 & 22 & 22 & 22 \\
\hline$E_{k}$ & $7.3 \mathrm{e}-10$ & $6.9 \mathrm{e}-10$ & $8.8 e-10$ & $6.3 \mathrm{e}-10$ & $5.0 \mathrm{e}-10$ \\
\hline CPU times & 0.44 & 1.23 & 2.22 & 4.33 & 7.34 \\
\hline
\end{tabular}

Example 5.2. Let $M=\operatorname{tridiag}(-1,2,-1) \in \mathbb{R}^{n \times n}$ and $N=\operatorname{tridiag}(0.5,0,0.5)$ $\in \mathbb{R}^{n \times n}$. For every $r \in \mathbb{R}$ we define the matrix $T_{r}$ as $T_{r}=M+r N$. Consider the following coupled matrix equations

$$
\left\{\begin{array}{l}
A_{11} X_{1}+X_{2} B_{12}=C_{1} \\
A_{21} X_{1}+X_{2} B_{22}=C_{2}
\end{array}\right.
$$

where $A_{11}=T_{4}, B_{12}=T_{4}, A_{21}=T_{3}$ and $B_{22}=T_{3}$. The right-hand matrices $C_{1}$ and $C_{2}$ are selected such that $\left(X_{1}^{*}, X_{2}^{*}\right)$ is the solution of (18) where $X_{1}^{*}$ and $X_{2}^{*}$ are the symmetric arrowhead matrices given by

$$
X_{1}^{*}=\left[\begin{array}{ccccc}
1 & 1 & 2 & \cdots & n-1 \\
1 & 2 & 0 & \cdots & 0 \\
2 & 0 & 3 & \cdots & 0 \\
\vdots & \vdots & \vdots & \ddots & \vdots \\
n-1 & 0 & 0 & \cdots & n
\end{array}\right], X_{2}^{*}=\left[\begin{array}{ccccc}
-1 & 1 & 2 & \cdots & n-1 \\
1 & -2 & 0 & \cdots & 0 \\
2 & 0 & -3 & \cdots & 0 \\
\vdots & \vdots & \vdots & \ddots & \vdots \\
n-1 & 0 & 0 & \cdots & -n
\end{array}\right] .
$$

The application of Algorithm 2 is examined to compute a symmetric arrowhead solution to the system (18). We utilize $\left(X_{1}(0), X_{2}(0)\right)=(0,0)$ as the initial guess and

$$
E_{k}=\max \left\{\frac{\left\|R_{1}(k)\right\|_{\infty}}{\left\|R_{1}(0)\right\|_{\infty}}, \frac{\left\|R_{2}(k)\right\|_{\infty}}{\left\|R_{2}(0)\right\|_{\infty}}\right\}<10^{-9}
$$


TABLE 2. Numerical results for Example 5.2, system (18).

\begin{tabular}{lccccc}
\hline & $n=1000$ & $n=2000$ & $n=3000$ & $n=4000$ & $n=5000$ \\
\hline \hline Iters & 45 & 48 & 50 & 47 & 48 \\
$E_{k}$ & $4.6 \mathrm{e}-10$ & $5.8 \mathrm{e}-10$ & $4.8 \mathrm{e}-10$ & $4.8 \mathrm{e}-10$ & $4.2 \mathrm{e}-10$ \\
CPU times & 1.61 & 5.75 & 13.59 & 21.77 & 33.95 \\
\hline \hline
\end{tabular}

TABLE 3. Numerical results for Example 5.2, system (20).

\begin{tabular}{lccccc}
\hline & $n=1000$ & $n=2000$ & $n=3000$ & $n=4000$ & $n=5000$ \\
\hline \hline Iters & 105 & 105 & 108 & 108 & 114 \\
$E_{k}$ & $8.4 \mathrm{e}-10$ & $8.4 \mathrm{e}-10$ & $8.7 \mathrm{e}-10$ & $8.5 \mathrm{e}-10$ & $7.7 \mathrm{e}-10$ \\
CPU times & 4.50 & 13.92 & 30.36 & 52.56 & 85.13 \\
\hline \hline
\end{tabular}

as the stopping criterion where $R(k)=\left(R_{1}(k), R_{2}(k)\right)$. Numerical results for different values on $n$ are given in Table 2. All the assumptions are as the previous example. Now consider the next coupled matrix equations

$$
\left\{\begin{array}{l}
A_{11} X_{1} B_{11}+A_{12} X_{2} B_{12}=C_{1} \\
A_{21} X_{1} B_{21}+A_{22} X_{2} B_{22}=C_{2}
\end{array}\right.
$$

where $A_{11}=A_{12}=T_{3}, A_{21}=A_{22}=T_{1}, B_{11}=B_{12}=T_{7}$ and $B_{21}=$ $B_{22}=8$. Numerical results are reported in Table 3 . We point here that all the assumptions are as the first part of this example. As seen, the handled algorithm is suitable.

Example 5.3. This example is given to verify the validity of the presented results in Subsection 4.3. To do this, we consider the system

$$
\left\{\begin{array}{l}
A_{11} X_{1} B_{11}+A_{12} X_{2} B_{12}=C_{1} \\
A_{21} X_{1} B_{21}+A_{22} X_{2} B_{22}=C_{2}
\end{array}\right.
$$

where

$$
\begin{gathered}
A_{11}=\left[\begin{array}{ccc}
4 & 2 & 0 \\
1 & 2 & 2 \\
0 & -1 & 3
\end{array}\right], \quad B_{11}=\left[\begin{array}{ccc}
2 & 0 & -1 \\
1 & 2 & 1 \\
1 & -1 & 3
\end{array}\right], \quad A_{12}=\left[\begin{array}{ccc}
-1 & 2 & 1 \\
1 & -2 & 2 \\
0 & 2 & 1
\end{array}\right], \\
B_{12}=\left[\begin{array}{ccc}
4 & 2 & 3 \\
0 & 3 & -2 \\
1 & 1 & -1
\end{array}\right], A_{21}=\left[\begin{array}{ccc}
2 & 3 & -1 \\
1 & 3 & 1 \\
0 & 1 & -2
\end{array}\right], \quad B_{21}=\left[\begin{array}{ccc}
1 & 3 & -1 \\
1 & 0 & -1 \\
-1 & 2 & 2
\end{array}\right], \\
A_{22}=\left[\begin{array}{ccc}
1 & -3 & 1 \\
1 & 3 & 0 \\
2 & 0 & 1
\end{array}\right], \quad B_{22}=\left[\begin{array}{ccc}
7 & -2 & 2 \\
1 & -3 & 1 \\
1 & 1 & -6
\end{array}\right],
\end{gathered}
$$


with the right-hand side matrices

$$
C_{1}=\left[\begin{array}{ccc}
31 & 18 & 24 \\
51 & 7 & 39 \\
34 & 8 & 23
\end{array}\right], \quad C_{2}=\left[\begin{array}{ccc}
22 & 25 & -28 \\
59 & -3 & 4 \\
68 & -38 & -23
\end{array}\right]
$$

It is not difficult to see that Eq. (21) has a unique symmetric arrowhead solution (see [1]) of the form $\left(X_{1}^{*}, X_{2}^{*}\right)$ where

$$
X_{1}^{*}=\left[\begin{array}{lll}
1 & 1 & 2 \\
1 & 2 & 0 \\
2 & 0 & 3
\end{array}\right], \quad X_{2}^{*}=\left[\begin{array}{lll}
3 & 1 & 2 \\
1 & 2 & 0 \\
2 & 0 & 1
\end{array}\right]
$$

An initial guess $(X(0), Y(0))=(0,0)$ and the stopping criterion $E_{k}<10^{-4}$ are exploited where $E_{k}$ is defined in (19). The approximate solution $X(56)=$ $\left(X_{1}(56), X_{2}(56)\right)$ is provided by Algorithm 2 where

$$
X_{1}(56)=\left[\begin{array}{ccc}
1.0002 & 1.0004 & 1.9996 \\
1.0004 & 1.9994 & 0 \\
1.9996 & 0 & 3.0003
\end{array}\right], X_{2}(56)=\left[\begin{array}{ccc}
2.9999 & 1.0001 & 2.0002 \\
1.0001 & 2.0000 & 0 \\
2.0002 & 0 & 0.9988
\end{array}\right] \text {. }
$$

We now consider

$$
\tilde{X}_{1}=\left[\begin{array}{ccc}
4 & 4 & 3 \\
5 & 3 & 0 \\
4 & -1 & 4
\end{array}\right], \quad \tilde{X}_{2}=\left[\begin{array}{ccc}
3 & 4 & 4 \\
4 & 2 & 5 \\
5 & -2 & 4
\end{array}\right]
$$

The matrix group $W_{1}=\left(W_{1}^{(1)}, W_{1}^{(2)}\right)$, defined in Subsection 4.3, is given by

$$
W_{1}^{(1)}=\left[\begin{array}{ccc}
4 & 4.5 & 3.5 \\
4.5 & 3 & 0 \\
3.5 & 0 & 4
\end{array}\right], \quad W_{1}^{(2)}=\left[\begin{array}{ccc}
3 & 4 & 4.5 \\
4 & 2 & 0 \\
4.5 & 0 & 4
\end{array}\right]
$$

Therefore, we have $\hat{C}=C-\mathcal{M}\left(W_{1}\right)=\left(\hat{C}_{1}, \hat{C}_{2}\right)$ where

$$
\hat{C}_{1}=\left[\begin{array}{ccc}
-94.5 & -34.5 & -46 \\
-39.5 & -23 & 14.5 \\
-41 & -15 & -29.5
\end{array}\right], \quad \hat{C}_{2}=\left[\begin{array}{ccc}
14 & -58.5 & 64 \\
-87.5 & -25.5 & 10.5 \\
-35 & 17.5 & 42.5
\end{array}\right] \text {. }
$$

In this case, the provided approximate solution of Eq. (16) by Algorithm 2 is $Y(44)=\left(Y_{1}(44), Y_{2}(44)\right)$ where

$$
\begin{aligned}
& Y_{1}(44)=\left[\begin{array}{ccc}
-2.9989 & -3.5011 & -1.5001 \\
-3.5011 & -0.9977 & 0 \\
-1.5001 & 0 & -0.9996
\end{array}\right], \\
& Y_{2}(44)=\left[\begin{array}{ccc}
-0.0004 & -3.0000 & -2.4999 \\
-3.0000 & 0.0002 & 0 \\
-2.4999 & 0 & -3.0008
\end{array}\right] .
\end{aligned}
$$


Hence

$$
\begin{aligned}
& X_{1}(44)=W_{1}^{(1)}+Y_{1}(44)=\left[\begin{array}{ccc}
1.0011 & 0.9989 & 1.9999 \\
0.9989 & 2.0023 & 0 \\
1.9999 & 0 & 3.0004
\end{array}\right], \\
& X_{2}(44)=W_{1}^{(2)}+Y_{2}(44)=\left[\begin{array}{ccc}
2.9996 & 1.0000 & 2.0001 \\
1.0000 & 2.0002 & 0 \\
2.0001 & 0 & 0.9992
\end{array}\right] .
\end{aligned}
$$

As seen the proposed algorithm has obtained a suitable solution for Problem 2.3 .

\section{Conclusion}

Recently, Li et al. [21] have derived the general expression for the least norm symmetric arrowhead solution of the least squares problem corresponding to the matrix equation $A X B+C Y D=E$ by exploiting the Moore-Penrose inverse and the Kronecker product. In this paper, an efficient iterative algorithm has been proposed to solve the least squares problem associated with a general class of coupled matrix equations over symmetric arrowhead matrices. More precisely, the conjugate gradient least squares (CGLS) method has been extended to construct the offered algorithm which obtains the symmetric arrowhead solution group of the considered problem within finite number of iteration steps for any initial symmetric arrowhead matrix group in the absence of roundoff errors. In particular, the least norm symmetric arrowhead solution group of the mentioned least squares problem can be computed by choosing an appropriate initial symmetric arrowhead matrix group. Scrutinized numerical experiments have confirmed the validity of the established theoretical results and reveal the good performance of the handled algorithm. The superior features of our examined algorithm over the existing manner [21] are that it is very simple, neat and inexpensive to handle for large an sparse problems. Whereas, the propounded algorithm outperforms Li et al.'s approach even for small size problems as examined for several examples.

\section{Appendix A}

The proof of Theorem 4.2. Without loss of generality, we only need to establish the validity of the assertions for $1 \leq i<j \leq k$. To this end, the mathematical induction is exploited. In what follows, for simplicity, we set

$$
\alpha_{k}=\frac{\|P(k)\|^{2}}{\|\mathcal{M}(Q(k))\|^{2}}, \quad \beta_{k}=\frac{\|P(k+1)\|^{2}}{\|P(k)\|^{2}} .
$$

Step 1. For $k=1$, we have

$$
\begin{aligned}
\langle P(0), P(1)\rangle & =\left\langle P(0), P(0)-\alpha_{0} \mathcal{A}\left(\mathcal{M}^{*}(\mathcal{M}(Q(0)))\right)\right\rangle \\
& =\|P(0)\|^{2}-\alpha_{0}\left\langle P(0), \mathcal{M}^{*}(\mathcal{M}(Q(0)))\right\rangle
\end{aligned}
$$




$$
\begin{aligned}
& =\|P(0)\|^{2}-\alpha_{0}\langle\mathcal{M}(Q(0)), \mathcal{M}(Q(0))\rangle \\
& =0 .
\end{aligned}
$$

Also, it can be seen that

$$
\begin{aligned}
\langle\mathcal{M}(Q(0)), \mathcal{M}(Q(1))\rangle & =\beta_{0}\|\mathcal{M}(Q(0))\|^{2}+\langle\mathcal{M}(Q(0)), \mathcal{M}(P(1))\rangle \\
& =\beta_{0}\|\mathcal{M}(Q(0))\|^{2}+\frac{1}{\alpha_{0}}\left\langle\mathcal{M}^{*}(R(0)-R(1)), P(1)\right\rangle \\
& =\beta_{0}\|\mathcal{M}(Q(0))\|^{2}+\frac{1}{\alpha_{0}}\left\langle\mathcal{A}\left(\mathcal{M}^{*}(R(0)-R(1))\right), P(1)\right\rangle \\
& =\beta_{0}\|\mathcal{M}(Q(0))\|^{2}+\frac{1}{\alpha_{0}}\langle P(0)-P(1), P(1)\rangle \\
& =\beta_{0}\|\mathcal{M}(Q(0))\|^{2}-\frac{1}{\alpha_{0}}\langle P(1), P(1)\rangle \\
& =0 .
\end{aligned}
$$

As $Q(0)=P(0)$ we conclude that

$$
\langle Q(0), P(1)\rangle=\langle P(0), P(1)\rangle=0 .
$$

Step 2. Suppose that the conclusions are true when $k=\ell$, i.e., the following relations hold for $i<l$,

$$
\langle P(i), P(l)\rangle=0, \quad\langle\mathcal{M}(Q(i)), \mathcal{M}(Q(l))\rangle=0 \quad \text { and } \quad\langle Q(i), P(l)\rangle=0 .
$$

We demonstrate that the validity of the assertions for $k=\ell+1$. It can be seen that

$$
\begin{aligned}
\langle P(l+1), P(l)\rangle & =\left\langle P(l)-\alpha_{l} \mathcal{A}\left(\mathcal{M}^{*}(\mathcal{M}(Q(l)))\right), P(l)\right\rangle \\
& =\langle P(l), P(l)\rangle-\alpha_{l}\langle\mathcal{M}(P(l)), \mathcal{M}(Q(l))\rangle \\
& =\langle P(l), P(l)\rangle-\alpha_{l}\left\langle\mathcal{M}(Q(l))-\beta_{l-1} \mathcal{M}(Q(l-1)), \mathcal{M}(Q(l))\right\rangle \\
& =\langle P(l), P(l)\rangle-\alpha_{l}\langle\mathcal{M}(Q(l)), \mathcal{M}(Q(l))\rangle \\
& =0 .
\end{aligned}
$$

Moreover by some straightforward computations and Proposition 3.2, we have

$$
\begin{aligned}
\langle\mathcal{M}(Q(l+1)), \mathcal{M}(Q(l))\rangle= & \left\langle\mathcal{M}(P(l+1))+\beta_{l} \mathcal{M}(Q(l)), \mathcal{M}(Q(l))\right\rangle \\
= & \langle\mathcal{M}(P(l+1)), \mathcal{M}(Q(l))\rangle+\beta_{l}\langle\mathcal{M}(Q(l), \mathcal{M}(Q(l))\rangle \\
= & \frac{1}{\alpha_{l}}\langle\mathcal{M}(P(l+1)), R(l)-R(l+1)\rangle \\
& +\beta_{l}\langle\mathcal{M}(Q(l)), \mathcal{M}(Q(l))\rangle \\
= & -\frac{1}{\alpha_{l}}\langle P(l+1), P(l+1)\rangle+\beta_{l}\langle\mathcal{M}(Q(l), \mathcal{M}(Q(l))\rangle \\
= & 0 .
\end{aligned}
$$

Note that $Q(l-1)$ is a symmetric arrowhead matrix group. In view of Proposition 3.2 , it is not difficult to conclude that

$$
\langle P(l+1), Q(l)\rangle=\left\langle P(l+1), P(l)+\beta_{l-1} Q(l-1)\right\rangle
$$




$$
\begin{aligned}
& =\beta_{l-1}\langle P(l+1), Q(l-1)\rangle \\
& =\beta_{l-1}\left\langle P(l)-\alpha_{l} \mathcal{A}\left(\mathcal{M}^{*}(\mathcal{M}(Q(l)))\right), Q(l-1)\right\rangle \\
& =-\alpha_{l} \beta_{l-1}\langle\mathcal{M}(Q(l)), \mathcal{M}(Q(l-1))\rangle \\
& =0 .
\end{aligned}
$$

For $j=1,2, \ldots, l-1$, by using the above results, we have:

$$
\begin{aligned}
\langle P(l+1), P(j)\rangle & =\left\langle P(l)-\alpha_{l} \mathcal{A}\left(\mathcal{M}^{*}(\mathcal{M}(Q(l)))\right), P(j)\right\rangle \\
& =\langle P(l), P(j)\rangle-\alpha_{l}\langle\mathcal{M}(Q(l)), \mathcal{M}(P(j))\rangle \\
& =-\alpha_{l}\left\langle\mathcal{M}(Q(l)), \mathcal{M}(Q(j))-\beta_{j-1} \mathcal{M}(Q(j-1))\right\rangle \\
& =0 .
\end{aligned}
$$

From Proposition 3.2, it is seen that

$$
\begin{aligned}
\langle\mathcal{M}(Q(l+1)), \mathcal{M}(Q(j))\rangle & =\left\langle\mathcal{M}(P(l+1))+\beta_{l} \mathcal{M}(Q(l)), \mathcal{M}(Q(j))\right\rangle \\
& =\langle\mathcal{M}(P(l+1)), \mathcal{M}(Q(j))\rangle \\
& =\frac{1}{\alpha_{j}}\langle\mathcal{M}(P(l+1)), R(j)-R(j+1)\rangle \\
& =\frac{1}{\alpha_{j}}\left\langle P(l+1), \mathcal{M}^{*}(R(j)-R(j+1))\right\rangle \\
& =\frac{1}{\alpha_{j}}\langle P(l+1), P(j)-P(j+1)\rangle \\
& =0 .
\end{aligned}
$$

Invoking the fact that $Q(j)$ is a symmetric arrowhead matrix group for each $j$, we derive:

$$
\begin{aligned}
\langle P(l+1), Q(j)\rangle & =\left\langle P(l)-\alpha_{l} \mathcal{A}\left(\mathcal{M}^{*}(\mathcal{M}(Q(l)))\right), Q(j)\right\rangle \\
& =\langle P(l), Q(j)\rangle-\alpha_{l}\langle\mathcal{M}(Q(l)), \mathcal{M}(Q(j))\rangle \\
& =0 .
\end{aligned}
$$

From Steps 1 and 2, the proof is competed by the principle of the mathematical induction.

Acknowledgments. The authors would like to express their sincere gratitude to anonymous referees for their valuable comments and suggestions.

\section{References}

[1] F. P. A. Beik and D. K. Salkuyeh, On the global Krylov subspace methods for solving general coupled matrix equation, Comput. Math. Appl. 62 (2011), no. 12, 4605-4613.

[2] — The coupled Sylvester-transpose matrix equations over generalized centrosymmetric matrices, Int. J. Comput. Math. 90 (2013), no. 7, 1546-1566.

[3] D. S. Bernstein, Matrix Mathematics: theory, facts, and formulas, Second edition, Princeton University Press, 2009.

[4] A. Björck, Numerical Methods for Least Squares Problems, SIAM, Philadelphia, 1996.

[5] C. F. Borges, R. Frezza, and W. B. Gragg, Some inverse eigenproblems for Jacobi and arrow matrices, Numer. Linear Algebra Appl. 2 (1995), no. 3, 195-203. 
[6] A. Bouhamidi and K. Jbilou, A note on the numerical approximate solutions for generalized Sylvester matrix equations with applications, Appl. Math. Comput. 206 (2008), no. 2, 687-694.

[7] M. Dehghan and M. Hajarian, An iterative algorithm for solving a pair of matrix equation $A Y B=E, C Y D=F$ over generalized centro-symmetric matrices, Comput. Math. Appl. 56 (2008), no. 12, 3246-3260.

[8] - The general coupled matrix equations over generalized bisymmetric matrices, Linear Algebra Appl. 432 (2010), no. 6, 1531-1552.

[9] _ Analysis of an iterative algorithm to solve the generalized coupled Sylvester matrix equations, Appl. Math. Model. 35 (2011), no. 7, 3285-3300.

[10] F. Ding and T. Chen, Gradient based iterative algorithms for solving a class of matrix equations, IEEE Trans. Automat. Control 50 (2005), no. 8, 1216-1221.

[11] - On iterative solutions of general coupled matrix equations, SIAM J. Control Optim. 44 (2006), no. 6, 2269-2284.

[12] F. Ding, P. X. Liu, and J. Ding, Iterative solutions of the generalized Sylvester matrix equations by using the hierarchical identification principle, Appl. Math. Comput. 197 (2008), no. 1, 41-50.

[13] A. El Guennouni, K. Jbilou, and A. J. Riquet, Block Krylov subspace methods for solving large Sylvester equations, Numer. Algorithms 29 (2002), no. 1-3, 75-96.

[14] D. Fisher, G. Golub, O. Hald, C. Leiva, and O. Widlund, On Fourier-Toeplitz methods for separable elliptic problems, Math. Comp. 28 (1974), 349-368.

[15] M. Hajarian, Developing the CGLS algorithm for the least squares solutions of the general coupled matrix equations, Math. Methods Appl. Sci. 37 (2014), no. 17, 27822798.

[16] M. Hajarian and M. Dehghan, The generalized centro-symmetric and least squares generalized centro-symmetric solutions of the matrix equation $A Y B+C Y^{T} D=E$, Math. Methods Appl. Sci. 34 (2011), no. 13, 1562-1579.

[17] D. Y. Hu and L. Reichel, Krylov-subspace methods for the Sylvester equation, Linear Algebra Appl. 172 (1992), 283-313.

[18] G. X. Huang, F. Ying, and K. Gua, An iterative method for skew-symmetric solution and the optimal approximate solution of the matrix equation $A X B=C$, J. Comput. Appl. Math. 212 (2008), no. 2, 231-244.

[19] K. Jbilou and A. J. Riquet, Projection methods for large Lyapunov matrix equations, Linear Algebra Appl. 415 (2006), no. 2, 344-358.

[20] T. Jiang and M. Wei, On solutions of the matrix equations $X-A X B=C$ and $X-$ $A \bar{X} B=C$, Linear Algebra Appl. 367 (2003), 225-233.

[21] H. Li, Z. Gao, and D. Zhao, Least squares solutions of the matrix equation $A X B+$ $C Y D=E$ with the least norm for symmetric arrowhead matrices, Appl. Math. Comput. 226 (2014), 719-724.

[22] J. F. Li, X. Y. Hu, X.-F. Duan, and L. Zhang, Iterative method for mirror-symmetric solution of matrix equation $A X B+C Y D=E$, Bull. Iranian Math. Soc. 36 (2010), no. $2,35-55$.

[23] D. P. O'leary and G. Stewart, Computing the eigenvalues and eigenvectors of symmetric arrowhead matrices, J. Comput. Phys. 90 (1990), no. 2, 497-405.

[24] B. Parlett, The Symmetric Eigenvalue Problem, Prentice-Hall, Englewood Cilffs, 1980.

[25] B. Parlett and B. Nour-Omid, The use of a refined error bound when updating eigenvalues of tridiagonals, Linear Algebra Appl. 68 (1985), 179-219.

[26] Z. H. Peng, The reflexive least squares solutions of the matrix equation $A_{1} X_{1} B_{1}+$ $A_{2} X_{2} B_{2}+\cdots+A_{\ell} X_{\ell} B_{\ell}=C$ with a submatrix constraint, Numer. Algorithms 64 (2013), no. 3, 455-480.

[27] Z. H. Peng, X. Y. Hu, and L. Zhang, Two inverse eigenvalue problems for a special kind of matrices, Linear Algebra Appl. 416 (2006), no. 2, 336-347. 
[28] The bisymmetric solutions of the matrix equation $A_{1} X_{1} B_{1}+A_{2} X_{2} B_{2}+\cdots+$ $A_{\ell} X_{\ell} B_{\ell}=C$ and its optimal approximation, Linear Algebra Appl. 426 (2007), no. 2, $583-595$.

[29] Z. H. Peng and J. Liu, The generalized bisymmetric solutions of the matrix equation $A_{1} X_{1} B_{1}+A_{2} X_{2} B_{2}+\cdots+A_{\ell} X_{\ell} B_{\ell}=C$ and its optimal approximation, Numer. Algorithms 50 (2009), no. 2, 127-144.

[30] Z. H. Peng and H. Xin, The reflexive least squares solutions of the general coupled matrix equations with a submatrix constraint, Appl. Math. Comput. 225 (2013), 425-445.

[31] Z. H. Peng and Z. J. Zhou, An efficient algorithm for the submatrix constraint of the matrix equation $A_{1} X_{1} B_{1}+A_{2} X_{2} B_{2}+\cdots+A_{\ell} X_{\ell} B_{\ell}=C$, Int. J. Comput. Math. 89 (2012), no. 12, 1641-1662.

[32] M. Robbé and M. Sadkane, A convergence analysis of GMRES and FOM methods for Sylvester equations, Numer. Algorithms 30 (2002), no. 1, 71-89.

[33] Y. Saad, Iterative Methods for Sparse Linear Systems, PWS Press, New York, 1995.

[34] D. K. Salkuyeh and F. P. A. Beik, An iterative method to solve symmetric positive definite matrix equations, Mathematical Reports 16 (2014), no. 2, 271-283.

[35] D. K. Salkuyeh and F. Toutounian, New approaches for solving large Sylvester equations, Appl. Math. Comput. 173 (2006), no. 1, 9-18.

[36] R. S. Wang, Functional Analysis and Optimization Theory, Beijing Univ of Aeronautics Astronautics, Beijing, 2003.

[37] A. G. Wu, G. Feng, G. R. Duan, and W. J. Wu, Finite iterative solutions to a class of complex matrix equations with conjugate and transpose of the unknowns, Math. Comput. Modelling 52 (2010), no. 9, 1463-1478.

[38] A. G. Wu, L. Lv, and G. R. Duan, Iterative algorithms for solving a class of complex conjugate and transpose matrix equations, Appl. Math. Comput. 217 (2011), no. 21, 8343-8353.

[39] A. G. Wu, B. Li, Y. Zhang, and G.-R. Duan, Finite iterative solutions to coupled Sylvester-conjugate matrix equations, Appl. Math. Model. 35 (2011), no. 3, 1065-1080.

[40] A. G. Wu, E. Zhang, and F. Liu, On closed-form solutions to the generalized Sylvesterconjugate matrix equation, Appl. Math. Comput. 218 (2012), no. 19, 9730-9741.

[41] Y. Yuan, Generalized inverse eigenvalue problems for symmetric arrow-head matrices, Int. J. Comput. Math. Sci. 4 (2010), no. 6, 268-271.

[42] H. Zha, A two-way chasing scheme for reducing a symmetric arrowhead matrix to tridiagonal form, J. Numer. Algebra Appl. 1 (1992), no. 1, 49-57.

[43] B. Zhou and G. R. Duan, On the generalized Sylvester mapping and matrix equation, Systems Control Lett. 57 (2008), no. 3, 200-208.

Fatemeh Panjeh Ali Beik

Department of Mathematics

VALI-E-ASR UNIVERSITY OF RAFSANJAN

RAFSANJAN, IRAN

E-mail address: f.beik@vru.ac.ir, beik.fatemeh@gmail.com

Davod Khojasteh Salkuyeh

Faculty of Mathematical Sciences

UNIVERSITY OF GUILAN

RASHT, IRAN

E-mail address: khojasteh@guilan.ac.ir, salkuyeh@gmail.com 